\title{
How do user-centered design studies contribute to cartography?
}

\author{
ROBERT ROTH
}

University of Wisconsin, Department of Geography, Madison, USA; e-mail: reroth@wisc.edu University of Twente, Department of Geo-Information Processing, International Training Center (ITC), http://orcid.org/0000-0003-1241-318X

ABSTRACT I ask in this essay: How do user-centered design studies contribute to cartography? Scholars in related fields increasingly recognize the intellectual value of employing user-centered processes to improve a single product and identify new design considerations for future products. To this end, I propose an analytical framework for organizing the contributions of user-centered design studies that includes eight opportunities for advancing cartography: (1) domain gap analyses, (2) adapted or novel user-centered methods, (3) streamlined user-centered design processes, (4) transferable design insights, (5) comprehensive user-centered design case studies, (6) novel or unique maps and visualizations, (7) summative controlled experiments, and (8) new insights into pressing geographic problems. I apply this framework against my own collaborative work in a retrospective analysis of three UCD case studies: the GeoVISTA CrimeViz visual analytics tool, the NOAA Lake Level Viewer climate change visualization, and the UW Cart Lab Global Madison mobile map.

KEY WORDS cartography - visualization - user-centered design - design studies - methodology - usability

ROTH, R. (2019): How do user-centered design studies contribute to cartography? Geografie, $124,2,133-161$.

Received November 2018, accepted May 2019.

CC Česká geografická společnost, z. s., 2019 


\section{Introduction: The case for user-centered design studies in cartography}

I ask in this essay: How do user-centered design studies contribute to cartography? Cartographers working towards higher degrees often feel faced with a critical decision at some point in their academic careers: do I want to make maps, or do I want to research maps? But, can and should we do both? What is the value of design-as-practice to cartographic research?

I argue that a user-centered approach offers one pathway for uniting research and practice in cartography. Scholars in the related fields of human-computer interaction, information visualization, and visual analytics increasingly recognize the intellectual value of user-centered processes that couple design and evaluation loops both to improve a single product and identify new design considerations for future products (e.g., Barkhuus, Rode 2007; Munzner 2009; Borkin et al. 2011; Lam et al. 2012; Isenberg et al. 2013). However, user-centered design studies mark an epistemological shift in the way we generate knowledge about maps and visualizations. In contrast to traditional quantitative experiments that simplify visual designs and control the testing environment to produce generalizable insights, design studies recruit target users and test in a realistic setting to reveal insights that are potentially transferable to other design contexts (Sedlmair, Meyer, Munzner 2012). However, many editorial boards and thesis committees do not consider such transferable design insights "good enough" to warrant publication.

In this essay, I extend our 2017 ICA (International Cartography Association) research agenda on users studies for interactive maps and visualizations (Roth et al. 2017) to advocate for the intellectual contributions of user-centered design studies in cartography. I begin by introducing user-centered design and its influences, highlighting characteristics that make user-centered design studies necessarily different from controlled experiments. Drawing on the Robinson et al. (2005) process, I then propose an analytical framework for organizing the scholarly contributions of user-centered design studies that includes eight opportunities for advancing cartography: (1) domain gap analyses, (2) adapted or novel user-centered methods, (3) streamlined user-centered design processes, (4) transferable design insights, (5) comprehensive user-centered design case studies, (6) novel or unique maps and visualizations, (7) summative controlled experiments, and (8) new insights into pressing geographic problems. Finally, I apply this framework in a retrospective analysis (following Sedlmair, Meyer, Munzner 2012) to three user-centered design case studies: the GeoVISTA CrimeViz visual analytics tool, the NOAA Lake Level Viewer climate change visualization, and the UW Cart Lab Global Madison mobile map. I conclude with final thoughts on integrating research and design in cartography. 


\section{Background: user-centered design and cartography}

User-centered design (henceforth UCD) describes a flexible, multiple-stage process for soliciting feedback from target users early and often during design (Norman 2013). Thus, UCD is not an equation prescribing all decisions a priori, but rather an iterative, mixed methodology for continuously evaluating and refining a design based on feedback from real rather than hypothetical target users. Specifically, UCD solicits user input to define and improve the product's usability (i.e., the ease of using the product, such as the learnability, memorability, efficiency of use, error frequency and severity, and subjective satisfaction) and utility (i.e., usefulness, or support for the identified user needs), two design characteristics often in tension with one another (e.g., Grinstein et al. 2003). UCD shares goals with ergonomics, user experience design, and usability engineering (Haklay, Nivala 2010), and was identified as one of four crosscutting challenges facing geovisualization in the 2001 ICA research agenda (MacEachren, Kraak 2001) preceding our 2017 agenda.

\subsection{UCD studies in practice}

On one hand: a user-centered approach informs cartographic design-as-practice. Project managers often plan complex maps and visualizations in development cycles, or milestone-driven and deadline-bound negotiations between concept (design) and implementation (development; e.g., v1.0, v2.0; Nielsen 1992). In an ideal dev cycle (Figure 1a), the implementation follows directly from the concept, with the initial design vision miraculously appearing in the final interactive. While an idealized, unilateral approach might yield creative, high-quality designs at times, it is far from a foolproof process. Instead, design typically is messy, and requires trial and error by even the most expert designers to puzzle through numerous design alternatives.

In contrast, a standard dev cycle (Figure 1b) often starts with implementation, with the product (and its failures and successes) then informing revisions and extensions. Many cartography agencies and companies follow this process, and there is valid reasoning for organizing around agile development of a minimum viable product (MVP), or a rapidly-created proof-of-concept that demonstrates core functionality, generates an early user base, and draws external investors (Tolochko 2016). However, a standard dev cycle often misses major usability issues, which can require substantial duplication of effort to resolve, and at worst can result in a perfectly usable product that does not support an actual user need, resulting in poor uptake and minimal use. 
Fig. 1 - Development cycles in practice: a an ideal dev cycle, $b$ - a standard dev cycle, c - a user-centered dev cycle

b)

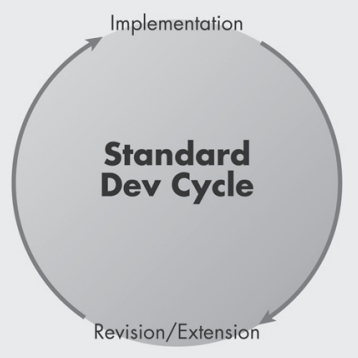

a)

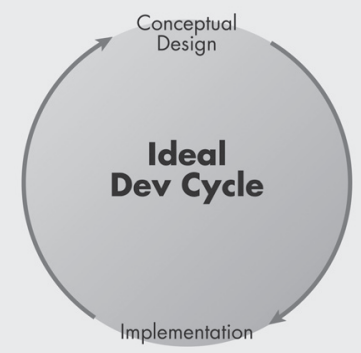

c)

User-Centered Dev Cycle

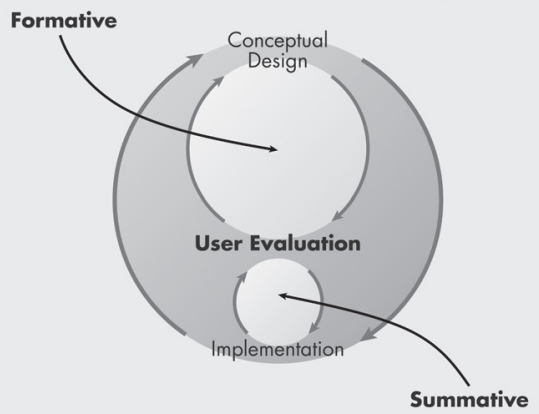

Finally, a user-centered dev cycle (Figure 1c) includes an evaluation stage with the target audience that mediates concept and implementation. A user-centered dev cycle also privileges early, formative user feedback over terminal, summative user feedback (Gabbard, Hix, Swan 1999), as fixing major issues is substantially more costly for fully-functional products than early prototypes. While common reasons for not following a user-centered dev cycle include perceived constraints on time, money, and expertise, a user-centered process instead saves resources by facilitating faster agreement about design decisions early in the project and avoiding unplanned revisions late in the project. To make efficient use of project resources, "discount" methods are employed to rapidly collect feedback from 3-5 target users at multiple stages in design and development (Nielsen 1994), with insights converging towards the final, successful design (Buttenfield 1999). 


\subsection{UCD studies in research}

On the other hand: UCD challenges the dominate approach to empirical research in cartography, a challenge that I argue opens rather than closes opportunities for user studies on maps and visualizations. As an enabling and interdisciplinary field, cartography draws from numerous methodological influences and embraces multiple ways of knowing. We chart cartography's diverse epistemological origins in the 2017 ICA research agenda, crossing influences from geography and psychology to information visualization and usability engineering. Across these methodological influences sits a central tension between results that are generalizable and reproducible and those that are transferrable and contextual.

Many expectations for sound cartographic research derive from our $20^{\text {th }}$ century relationship with psychology. Psychology as a "basic" or foundational science prescribes control in study design to establish generalizable insights that apply across use and user contexts. For cartography, this often results in a large sample size of non-target users (e.g., undergraduate students), map stimuli that are greatly simplified and use synthetic data, an experimental procedure reduced to simple benchmark tasks, and hypothesis testing on only a small set of experimental factors while controlling for all other aspects of design. Such control is needed to promote reproducible results, allowing scholars to review and critique experimental protocols, organize and compare study designs and outcomes, and ultimately implement past studies to validate generalizability of their results (Griffin, Çöltekin, Christophe 2018). While the results of psychology-driven user studies are narrow and shallow, they are ostensibly applicable across many-to-all design contexts and thus serve as baseline guidance for design-as-practice.

In contrast, new expectations for empirical research are emerging in the creative design branches of computer science, such as human-computer interaction, information visualization, and visual analytics. Advocates call for the legitimization of design studies that track the conceptualization, implementation, and evaluation of a product that meets a real-world problem domain and target user group (Munzner 2009). Compared to controlled experiments seeking generalizable insights, design studies seek transferable insights that directly improve a single product in question and that may be useful for similar design and development scenarios in the future. Such insights therefore are inherently contextual, and thus capture the design nuance for supporting a focused set of target users and needs (Griffin et al. 2017).

For cartography, a "user-centered" design study often includes a small sample size of target users recruited at multiple points in the dev cycle, complex and datadriven map designs ranging from early paper mockups to partially-functional prototypes, ecologically-valid use cases observed in real-world settings, adaptations of often qualitative social science methods for collecting feedback, and 
iterative consideration and evaluation of multiple design alternatives throughout the user-centered dev cycle, all to the end of producing a more usable and useful final product for a single design context (Ooms, Skarlatidou 2018). Design studies also can embrace critiques from digital geographies and other critical science and technology studies (see Crampton 2011; Elwood, Leszczynski 2018 for discussion), with a focus on transferability a potentially fruitful alternative for switching emphasis from product to process and, through this process, for finding contextual, situated, and more meaningful design insights (Kitchin, Dodge 2007). Accordingly, the results of UCD studies are wide and deep, but specific to a single use and user context.

\section{Framework: how user-centered design studies contribute to cartography}

\subsection{Contributions to design-as-practice}

In both research and practice, UCD studies emphasize process. Sedlmair, Meyer and Munzner (2012) offer a multi-stage process for conducting design studies, identifying three types of research contributions: (1) problem characterization and abstraction (early in the design process), (2) validation of visualization design (concluding the design process), and (3) reflection on the design study (after completing the design process). Thus, a design study encapsulates the entire process, not just the evaluation within the process. Further, a design study does not necessarily require empirical evaluation per se, but many design studies employ a usercentered approach and motivate empirical evidence to characterize the problem context (1) and validate the design (2); I expand upon these three "contributions" of UCD studies through my own retrospective analysis below (3).

There are a number of alternatives to the Sedlmair, Meyer and Munzner (2012) UCD process that elucidate additional ways that a UCD study can improve a single product and contribute new design insight to cartography. Many of these alternatives are specific to maps and visualizations (e.g., Gabbard, Hix, Swan 1999; van Elzakker, Wealands 2007; Tsou, Curran 2008; Haklay, Nivala 2010; Koh et al. 2011; Bruggmann, Fabrikant 2016). In my own research and design experience, I have found the process described by Robinson et al. (2005) particularly instructive for planning and executing a UCD study. Building on Slocum et al. (2003), Robinson et al. (2005) describe six, highly-iterative stages of UCD (Figure 2; terminology updated from Roth, Ross, MacEachren 2015):

1. Needs assessment: A needs assessment (also task analysis or work domain analysis) is an initial characterization of the target user needs and other relevant use context. In practice, a needs assessment formalizes user personas (i.e., generic descriptions of different kinds of target users) and use case scenarios 
Fig. 2 - The Robinson et al. (2005) usercentered design process

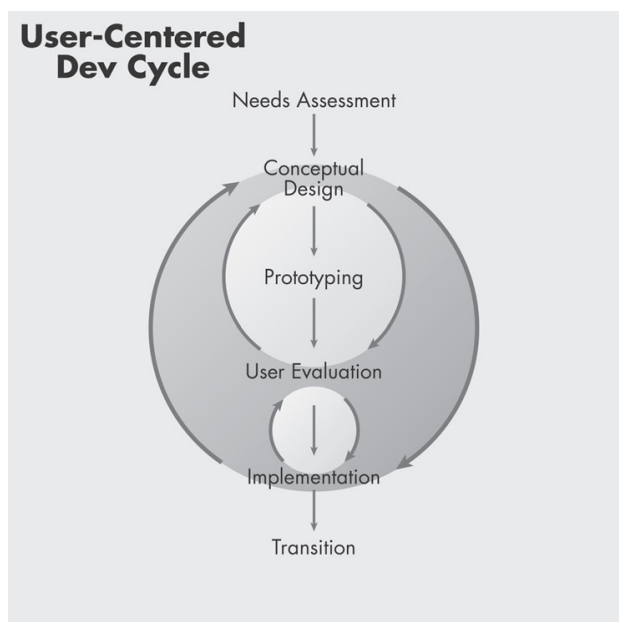

(i.e., "stories" about hypothetical use; Rosson, Carroll 2002). User personas and use case scenarios help designers keep in mind who the product is for (and not for). The needs assessment also answers a number of questions about the design context, such as user goals, currently met and unmet user needs, user backgrounds and abilities, and the users' technological infrastructure and environmental setting.

2. Conceptual design: Conceptual design then translates feedback from the needs assessment into design features for the proposed product. Sometimes described as a requirements analysis, conceptual design enumerates the functional requirements for the product, listing all necessary geospatial data, map representations, and user interactions as well as non-functional requirements such as accessibility, customization, and interoperability (Sluter, van Elzakker, Ivánová 2017). Conceptual design also may include a competitive analysis comparing functionality found in similar products to expose common practices and functional gaps (Nielsen 1992).

3. Prototyping: Conceptual design is followed by prototyping, or creation of visual descriptions of proposed functionality to illustrate the otherwise text-based list of requirements. In practice, prototypes begin as low-fidelity wireframes, perhaps as pen-and-paper sketches or simple visual outlines, and later include high-fidelity mockups using real datasets and comprehensive design specifications (Lloyd, Dykes 2011).

4. User evaluation: Following a user-centered approach, target users and other stakeholders evaluate prototypes of all degrees of fidelity on their prospective usability and utility. Formative evaluation of early prototypes identifies missing functionality, considers potential bottlenecks in navigation and map use, discusses the overall visual branding and style specifications, and reveals 
Table 1 - The Robinson et al. (2005) user-centered design process and associated design artifacts that are valuable from a practical standpoint

\begin{tabular}{ll}
\hline Stage & Design Artifacts / Practical Contributions \\
\hline Needs assessment & user personas, use case scenarios \\
Conceptual design & requirements document, competitive analysis \\
Prototyping & low-fidelity wireframes, high-fidelity wireframes \\
User evaluation & utility and usability feedback \\
Implementation & alpha release, beta release \\
Transition & edit sheet, issue log, final release \\
\hline
\end{tabular}

potential "head-slapper" usability issues before investing heavily in production (Krug 2000, p. 158). As described above, user evaluations need only include 3-5 target users, but are administered throughout the process to triangulate design insights. User evaluations draw from a wide range of empirical methods, and increasingly rely on qualitative feedback (see Štěrba et al. 2014; Roth, Ross, MacEachren 2015; van Elzakker et al. 2017; Ooms, Skarlatidou 2018 for recent reviews).

5. Implementation: The user-centered process then transitions from design to implementation, which might entail map production for static products like atlases and map development for interactive and digital products like web or mobile maps. In practice, the team works through a series of evaluation-implementation loops using milestone releases (e.g., v0.1, v0.2). In alpha releases, the interactive is partially functional and unstable, allowing developers to garner low-cost feedback on the general direction of development and to talk through tricky functionality. In beta releases, the interactive is fully functional, but still unstable, with summative evaluation used mostly to identify and resolve simple usability errors and programming bugs.

6. Transition: In the final transition, the product is sent for printing or deployed online. In practice, the transition is facilitated by an edit sheet or issue log to capture and track any final changes. The technical transition may terminate the project or initiate a new dev cycle; regardless, effort should be made to archive useful insight from the process (e.g., user requirements, spec sheets, code repositories) to inform future design (Buckingham, ed. 2019).

Table 1 lists each stage in the Robinson et al. (2005) UCD process and the associated design artifacts that are valuable from a practical standpoint. 


\subsection{Contributions to research scholarship}

While the Robinson et al. (2005) process has clear benefits for cartographic designas-practice, it also illuminates ways that UCD studies can generate profound design insights about cartography that are of similar intellectual merit to those produced through controlled experimentation. There are several critical (and in some cases, watershed) moments during a design study when designers can slow the process and enhance the overall contribution by either approaching a given stage somewhat differently or collecting additional user feedback beyond baseline UCD expectations (Figure 3). I argue there are at least eight such moments in UCD studies affording transferable, contextual contributions to cartography:

1. Domain gap analyses: A domain gap analysis characterizes current practices and unmet needs regarding the use of maps and visualizations (or other kinds of designs) in a specific problem context. The potential for a domain gap analysis exists at the nexus of the needs assessment and conceptual design stages (Figure 3a), where user needs, values, and experiences are translated

\section{UCD Study Contributions}

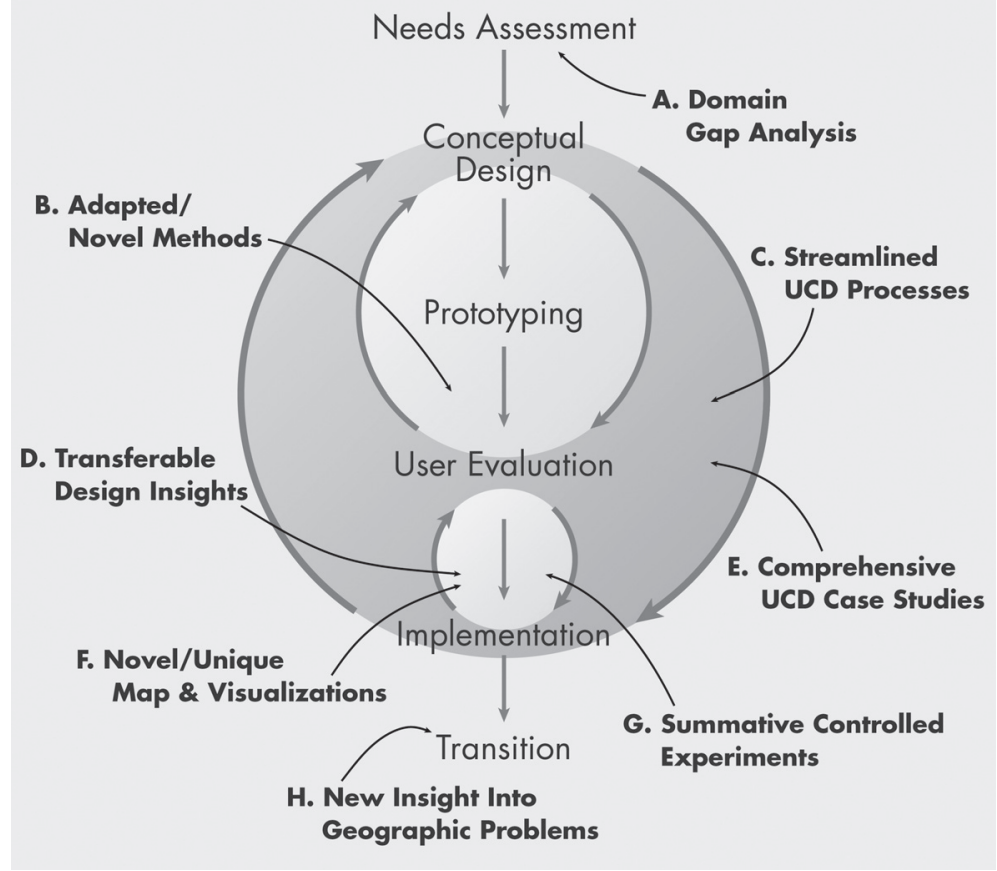

Fig. 3 - How user-centered design studies contribute: an analytical framework 
into functional and non-functional requirements. However, a gap analysis is wider in scope than the problem characterization typically employed by a design study, instead identifying persistent challenges and future directions crosscutting the problem domain, with perhaps only one challenge addressed by the developed product. Thus, a useful gap analysis surveys the current design and technology landscape within a problem domain, characterizes different user groups within this problem domain, captures variation in use contexts including institutional barriers to uptake, and charts a course for future research on maps and visualization for the specific domain (i.e., fills the "gaps"). A domain gap analysis is explicitly contextual, covering only one problem domain and not attempting to speak generally to all applications of maps and visualizations.

A key concern with a domain gap analysis embedded within a UCD study is participant representation in the needs assessment: can they speak for the entire problem domain? Accessing expert domain users is difficult, and in some cases, the population of experts is small. Further, user experiences vary across contexts, and capturing these differences in opinion is essential for a useful gap analysis. Accordingly, expanding the needs assessment to a gap analysis requires the designers to complete additional background reading and training to identify tensions within the domain, hone question sets, and recruit a representative sample. While time-consuming, this added scouring of secondary sources is a valuable, even enlightening preparatory step for the needs assessment that in itself can identify new ways of approaching design.

2. Adapted or novel methods: Inclusion of a user-centered evaluation (Figure 3b) affords the opportunity to explore adapted or novel empirical methods that produce transferable rather than generalizable insights. Importantly, the contribution is not just in running the adapted or novel method and reporting its results, but also in discussing the range and sensitivity of the method when transferred to other use and user contexts. Reflections on the experience with the adapted or novel method build towards new gold standards for administering user-centered evaluations, one of the pressing needs listed in the 2017 ICA research agenda.

A key concern with exploring adapted or novel methods within a UCD study is the small sample size recommended for any single evaluation within a user-centered dev cycle. While a discount sample of 3-5 target users may be sufficient and justifiable as a proof-of-concept for complex, participatory, and longitudinal methods, other methods seeking superficial but broad input (e.g., standardized surveys) require larger sample sizes and triangulation across multiple development cycles to warrant publication. Two additional strategies to strengthen the contribution include method articles that synthesize the use of an adapted or novel method across different design projects (e.g., Çöltekin et al. 
2009; Roth et al. 2011) - potentially comparing study design and execution for basic science versus UCD - and reanalysis articles that apply new measures and analyses on previously collected user input by way of assessing alternatives and breakpoints (e.g., Davies 1998; Klippel, Weaver, Robinson 2011).

3. Streamlined UCD processes: Another pressing need identified in the 2017 ICA research agenda is streamlining the UCD process for maps and visualizations, and in particular, adjusting where user evaluations may fall in the overall user-centered dev cycle (Figure 3c). Because most proposed UCD processes are highly iterative, numerous supplement, shortcut, and offshoot activities may be beneficial for specific use and user contexts as well as for different limitations on time, resources, technology, and expertise. For instance, Slocum et al. (2003) begin by developing rapid prototypes to inform user discussion while Bruggmann and Fabrikant (2016) alternate evaluations between users and designers to streamline product development. Such process contributions help designers and researchers better plan and manage a project given its unique opportunities and constraints.

A key concern of reporting streamlined and contextualized processes is validation within a single user-centered dev cycle. Processual contributions must clearly describe the reasoning behind the modification, relating workflow changes to unique aspects of the use and user context (i.e., justifying why a conventional UCD process could not work for the given product). Processual contributions also must weigh the pros and cons of the alternative process when transferred to other use and user contexts. Finally, processual contributions may include a hybrid of controlled experimentation and user-centered evaluations, where different teams follow different design processes and compare milestone deliverables to reflect on alternatives.

4. Transferable design insights: Seemingly obvious, but sometimes lost in the chaos of development, is archiving the valuable design revisions executed during prototype-evaluation and evaluation-implementation loops (Figure 3d). Design contributions summarize the "take-home points" or "lessons learned" - some controversial or unexpected - from the UCD process as illustrated by the final map or visualization. Reported insights also can include negative results (Kosara et al. 2003): designs that did not to work for the given use and user context based on user feedback, but that may inform design alternatives or pitfalls on other projects.

A key concern with presenting design contributions from UCD studies is claiming the insights as universal. Insights from design studies are necessarily transferable and contextual, meant not to impose generalized recommendations for design-as-practice but instead to stimulate new considerations for future projects. Thus, UCD studies float interesting ideas into the community, rather than declare rules for design. A narrative approach lends itself well 
to the reporting of transferable and contextual insights, putting the reader alongside the designer when explaining each evaluation-implementation loop. Figures illustrating before/after designs or providing an array of negative design alternatives can complement the design narrative, using the figure captions to bring additional first-person narration about design justification into the manuscript.

5. Comprehensive UCD case studies: A third pressing need from the 2017 ICA research agenda is the reporting of comprehensive UCD case studies. If reproducibility is not possible with UCD studies, given the focus on a single product and dev cycle, then perhaps provenance is the ultimate goal of design studies, enabling readers to track each stage in the UCD process and the constituent decisions therein (Figure 3e). Capturing the analytical provenance of users within maps and visualizations has received attention in cartography and related fields (e.g., Robinson, Weaver 2006). There is a growing, complementary interest in externalizing the thinking of designers throughout the process of design (Rooney et al. 2017), with provenance facilitated by development tools such as Github and Observable. Again following a narrative approach, the reconstruction of UCD case studies provide self-contained "design stories" that are useful beyond the functional lifetime of a given product, serve as instructional materials for education and training on UCD, and provide crucial success stories to promote buy-in and uptake of user-centered dev cycles.

A key concern with reporting a comprehensive UCD case study is sheer volume of materials and feedback collected through a user-centered dev cycle. It often is difficult to tell a complete design story within the $6,000-8,000$ word limits of most journals, and many of the details may appear rote to some reviewers. Accordingly, the cartography community needs to promote design provenance by establishing standards for consistency and comprehensiveness of study reporting. This may include new ways of utilizing supplemental materials in journals, or centralized repositories hosted outside of any single journal that archive all UCD study materials (White 2017).

6. Novel or unique maps and visualizations: The primary outcomes of UCD in practice are the implemented maps and visualizations themselves (Figure 3f). I have observed a tendency to discount a mapping product as a contribution in itself, both on thesis committees and in peer-reviewed journals. I see the debate both ways: On one side, cartography as a discipline builds upon the work of past designers and scholars, envisioning new pathways forward using insight from the collective. A map or visualization presented without design context or evidence of success may not be enough to nudge our collective forward. On the other side, and in considering cartography as much art as science, the product of design is the best glimpse into the maker's creative process, and leaving the map or visualization devoid of comment invites discourse and multiple interpretations. 
For UCD studies, excessive user evaluations actually may undermine creativity and innovation (Greenberg, Buxton 2008). Ultimately, I think there is space for presenting maps and visualization - as least ones that are novel or unique - as contributions in themselves, and there are now outlets specializing in such contributions including the Journal of Maps and the Visual Fields section of Cartographic Perspectives.

A key concern with publishing the product of a UCD study is continuous access. Many innovative maps and visualizations are proprietary, restricting access behind publisher costs or user accounts. Products that provide open access (open = gratis) may rely on restrictive proprietary technology, and even maps and visualizations that are truly open source (open = libre) often have a shelf life of just several years before deprecation for newer mapping technology. This is particularly a problem for interactive, online, and mobile maps: for instance, almost all of the interactive maps I developed as a graduate student no longer work, including the GeoVISTA CrimeViz tool discuss in the next section. If a map or visualization is to stand on its own as a scholarly contribution, then it must be freely available to all and fully available in 5, 25, and 100 years from publication for future generations to examine and critique. Accordingly, we need new ways to archive interactive, online, and mobile maps to ensure that we do not lose a generation of cartographic design innovation.

7. Summative controlled experiments: As introduced above, most UCD processes emphasize formative over summative user evaluations, as the cost of making major revisions on the beta or final release is prohibitively expensive (Figure $3 g$ ). However, the summative stage of evaluation offers an opportunity to administer a controlled experiment, going beyond validating the map or visualization and instead seeking generalizable insights into to a small set of thornier design questions exposed during the UCD process. A controlled experiment embedded within a UCD study overcomes one of the primary criticisms of basic science: convenience sample of a non-representative user group. It is common for the recruited target users to "buy-in" to the design and "take ownership" over the design by the final stages of a UCD process, as they see their feedback valued and utilized by the design team with each new release. Accordingly, the otherwise difficult to access but representative target users become motivated participants in a summative controlled experiment.

A key concern with attempting a controlled experiment at the end of a UCD process is the cost. The final release often requires simplification for summative evaluation to control for confounding variables, adding development time. Administering the controlled experiment also draws valuable time from both the designers and target users, time not spent towards improving the actual product. However, clients and target users may find these added costs an acceptable compromise for receiving a product tailored to their needs, particularly in 
university or non-profit settings where profit margins are less influential on resource allocation and collaborative partnership is expected.

8. New insights into pressing geographic problems: Perhaps the ultimate goal of a UCD study in both research and practice is creating a map or visualization that addresses pressing geographic problems (Figure $3 \mathrm{~h}$ ). Cartography is more than just a service or technology sold for profit to different problem domains. At their best, UCD studies facilitate good, resulting in maps and visualizations that enable their users to confront inequities, consider alternatives, and affect meaningful change. But, maps and visualizations also can harm, and sometimes even kill. UCD studies can expose the political economy, governance, and other power structuring within a problem domain, an important function of UCD studies highlighted in the 2017 ICA research agenda.

A key concern when contributing to pressing geographic problems through a UCD study is clarification of the relationship between researcher and subject, or designer and user. A successful UCD collaboration positions recruited users as equals in the design process, with user evaluations designed to elicit expertise rather than test knowledge. Thus, the user is a partner, not a subject, working with the designers towards a mutual goal. Projects that explicitly address geographic problems - particularly sensitive, power-laden, or other difficult contexts - can easily slip into a researcher-subject binary that is counterproductive to the goal of improving conditions and doing good.

Table 2 lists opportunities for UCD studies to contribute to research scholarship, identifying the type of contributions and associated concerns when planning and executing the study. The Figure 3 framework provides a rubric against which to review a UCD study as well as a foundation to consider additional ways that a UCD study might contribute to research scholarship. In the next section, I level the analytical framework against my own past collaborative work in a retrospective

Table 2 - Opportunities for a UCD study to contribute to research scholarship, with associated types of contributions and considerations therein

\begin{tabular}{lll}
\hline Opportunity & Type of Contribution & Key Concern \\
\hline Domain gap analyses & context & participant representation \\
Adapted / novel user-centered methods & method & small sample size \\
Streamlined UCD processes & process & validation from one dev cycle \\
Transferable design insights & design & overstatement of generalizability \\
Comprehensive UCD case studies & provenance & volume of materials and feedback \\
Novel / unique maps or visualizations & product & continuous access and archiving \\
Summative controlled experiments & basic science & cost \\
New insights into geographic problems & good & clarifying the researcher-subject \\
& & and designer-user relationship \\
\hline
\end{tabular}


analysis of three UCD case studies. I selected these case studies because they resulted in very different scholarly contributions to cartography (Table 3), despite drawing from the same UCD principles summarized above.

\section{Three case studies: a retrospective analysis}

\subsection{GeoVISTA CrimeViz}

GeoVISTA CrimeViz was an interactive and web-based visual analytics application supporting proactive and data-driven crime analysis and public safety (Figure 4). We designed the CrimeViz concept as a flexible set of tools extensible to any realtime, event-based data feed. We demonstrated the utility of the toolkit through a long-term, in-depth UCD study spanning 2009-2011 completed in collaboration between the Penn State GeoVISTA Center and the Harrisburg (Pennsylvania, U.S.) Bureau of Police. As a Penn State PhD student at the time, I had the opportunity of contributing to the client-side design and user evaluations as part of a large, multi-site development team. After the better part of a decade of use, GeoVISTA CrimeViz is now retired in favor of alternative mapping and analytics tools.

Looking back on my experience, the GeoVISTA CrimeViz UCD study resulted in a number of scholarly contributions following the Figure 3 framework. First, we established the in-depth collaboration with the Harrisburg Bureau of Police through a domain gap analysis. We began the UCD study by visiting seven intermediate- to large-size law enforcement agencies in the Northeastern United States, organizing current practices and unmet needs into six categories: (1) data, (2) representation, (3) interaction, (4) spatial analysis, (5) temporal analysis, and (6) use cases. The representation+interaction breakdown remains the way I organize functional needs on interactive mapping and visualization projects, with spatial and temporal analyses important additional categories in the context of visual analytics. The City of Harrisburg experienced fiscal duress during the 2007-2009 U.S. economic recession, and our site visit to the Harrisburg Bureau of Police in 2009 prompted follow-up conversations to create an easy-to-use mapping interface to make up for technology and expertise shortfalls caused by budget cuts. While uncommon, in this case the broader domain gap analysis lead directly into the focused needs assessment with the Harrisburg Bureau of Police (see Roth et al. 2013 for details).

We made use of common empirical methods across the GeoVISTA CrimeViz UCD study: interviews for the needs assessment (described above), think aloud on the alpha release, and online surveys on the beta and final releases. One small methodological contribution was development of a standardized, 20-question survey for soliciting rapid (e.g., 2-3 minutes) feedback on usability versus utility at multiple stages in a UCD study. I since used the standardized survey for rapid 
Table 3 - A retrospective analysis of scholarly contributions from three prior UCD case studies using the Figure 3 analytical framework

\begin{tabular}{|c|c|}
\hline Contribution & GeoVISTA CrimeViz \\
\hline Domain Gap Analyses & $\begin{array}{l}\checkmark \text { interviews organized by: } \\
\text { (1) data } \\
\text { (2) representation } \\
\text { (3) interaction } \\
\text { (4) spatial analysis } \\
\text { (5) temporal analysis } \\
\text { (6) use cases }\end{array}$ \\
\hline Adapted / Novel Methods & - novel usability/utility survey \\
\hline Streamlined UCD Processes & - user $\rightarrow$ utility $\rightarrow$ usability workflow \\
\hline Transferable Design Insights & $\begin{array}{l}\text { - speak user's language when referencing data categories } \\
\text { - hexagonal binning rather than spatial interpolation for big data } \\
\text { queries } \\
\text { - space, time, and attribute interface layout } \\
\text { - time wheel filtering interface } \\
\text { - shortcuts for common temporal queries }\end{array}$ \\
\hline Comprehensive UCD Case Studies & $\checkmark$ \\
\hline Novel / Unique Designs & $\checkmark$ archived source code and video demonstrations on project website \\
\hline Summative Controlled Experiments & $\begin{array}{l}\checkmark \text { generalizable insights include: } \\
\text { - cognitive sophistication of objectives increases from } \\
\text { identify } \rightarrow \text { compare } \rightarrow \text { rank } \rightarrow \text { associate } \rightarrow \text { delineate } \\
\text { - search vs. filter should be used for simple vs. complex objectives } \\
\text { - identification of suboptimal interaction operator/objective } \\
\text { combinations that should be prevented in interaction design }\end{array}$ \\
\hline $\begin{array}{l}\text { New Insight into Geographic } \\
\text { Problems }\end{array}$ & $\begin{array}{l}\text { - arsons peak on Wednesdays and follow a bell curve centered } \\
\text { midweek } \\
\text { - increased foot patrol leads to reduced violent crimes in summer } \\
\text { months }\end{array}$ \\
\hline
\end{tabular}

feedback on a number of UCD studies, including the NOAA Lake Level Viewer and Global Madison projects described below.

A major contribution from the GeoVISTA CrimeViz UCD study regarding process was clarification of prototype-evaluation and evaluation-implementation loops within the user-centered dev cycle. Given the iterative nature of UCD processes, it might appear that evaluation and revision is endless, with the final release perpetually delayed with one more evaluation-revision loop. We planned the GeoVISTA CrimeViz UCD process to negotiate a series of user $\rightarrow$ utility $\rightarrow$ usability loops, first determining user needs and characteristics, then setting or revising expected utility to respond to these user needs, and finally improving the usability as much 


\begin{tabular}{|c|c|}
\hline NOAA Lake Level Viewer & Global Madison \\
\hline $\begin{array}{l}\checkmark \text { competitive analysis organized by: } \\
\text { (1) waterline/flood extent (rep) } \\
\text { (2) uncertainty (rep) } \\
\text { (3) basemap/context (rep) } \\
\text { (4) operators (int) } \\
\text { (5) web mapping tech (int) }\end{array}$ & $\begin{array}{l}\checkmark \text { literature review organized by: } \\
\text { (1) map composition/layout (rep) } \\
\text { (2) scale/generalization (rep) } \\
\text { (3) projection (rep) } \\
\text { (4) symbolization (rep) } \\
\text { (5) typography (rep) } \\
\text { (6) map elements (rep) } \\
\text { (7) interaction }\end{array}$ \\
\hline $\begin{array}{l}\text { - cognitive walkthroughs with low- vs. high-fidelity } \\
\text { wireframes }\end{array}$ & - online survey + field observation combination \\
\hline $\begin{array}{l}\text { - separation of design and development } \\
\text { - linearize design stages }\end{array}$ & $\begin{array}{l}\text { - parallelize by storytelling vs. UI/UX vs. evaluation } \\
\text { teams }\end{array}$ \\
\hline $\begin{array}{l}\text { - exposed vs. inundated map design } \\
\text { - clarify entry point / first click } \\
\text { - separate configure vs. context interface controls } \\
\text { - evoke visual metaphor for enumeration vs. } \\
\text { inundation } \\
\text { - relate design to IPCC climate change scenarios } \\
\text { - include local context about impacted people and } \\
\text { places }\end{array}$ & $\begin{array}{l}\text { - focus on critical issues that leave user stranded } \\
\text { - append GPS with offline/static maps } \\
\text { - supply a consistent feed of information } \\
\text { - enforce associations between map and landscape } \\
\text { - promote user safety when mobile }\end{array}$ \\
\hline$\times$ & $\checkmark$ \\
\hline $\begin{array}{l}\sim \text { no source code archive/repository; } \pm 6 \mathrm{ft} \text { tilesets } \\
\text { available for reuse }\end{array}$ & $\begin{array}{l}\checkmark \text { archived source code on journal website as } \\
\quad \text { supplemental materials }\end{array}$ \\
\hline$x$ & $x$ \\
\hline$x$ & $\begin{array}{l}\text { - Madison's (WI, U.S.) relationships to global } \\
\text { inequities, interdependencies, and alternatives. } \\
\text { - situated learning }\end{array}$ \\
\hline
\end{tabular}

as possible given the utility threshold. We worked through three such loops for GeoVISTA CrimeViz, with the alpha, beta, and final releases the milestones for each loop.

Through the GeoVISTA CrimeViz UCD process, we identified a number of potentially transferable design insights. We learned early that the application needed to "speak the user's language" (Molich, Nielsen 1990, p. 339), drawing on an inhouse numbering scheme for referencing different types of crime. We also learned that direct aggregation to hexagonal bins, rather than spatial interpolation into "hot spot" or "heat" maps, was the preferred representation solution for big data queries, as interpolation could result in misleading spatial clusters in locations 


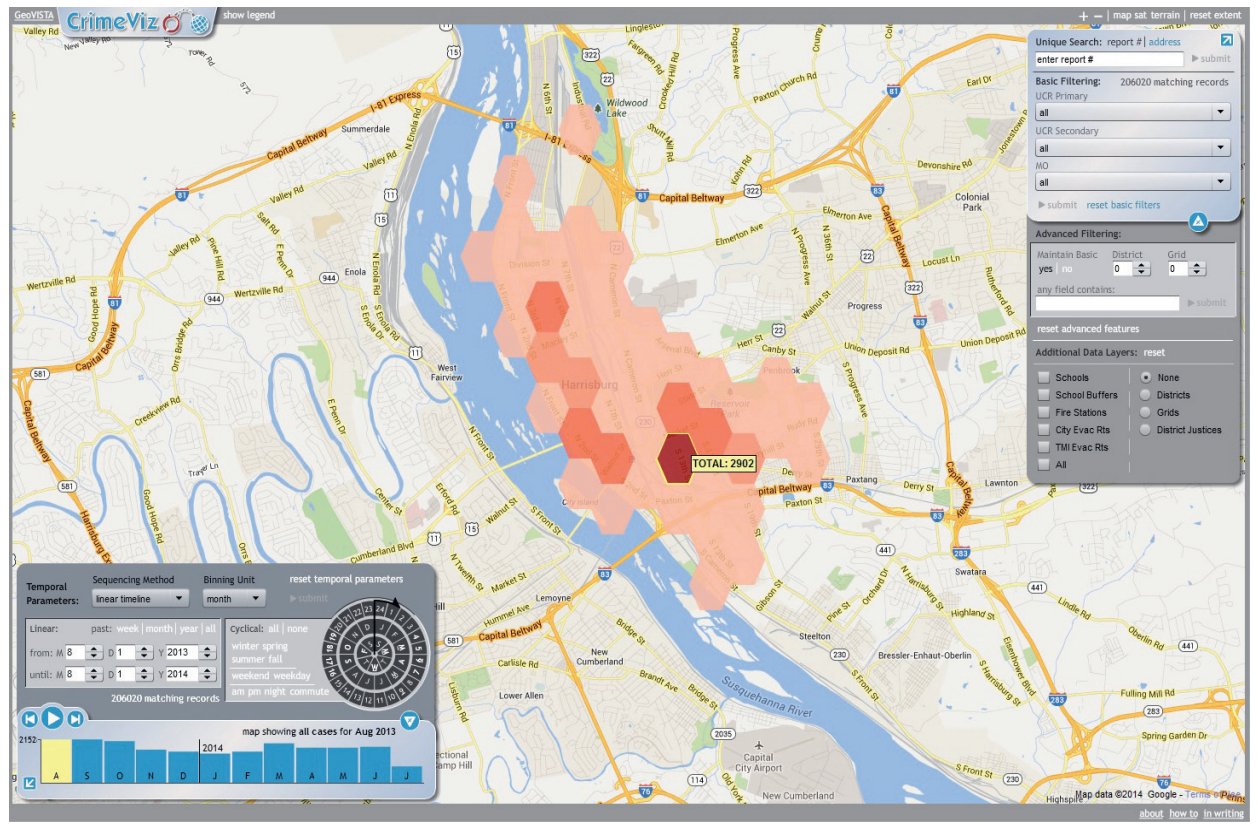

Fig. 4 - GeoVISTA CrimeViz. Source: http://www.geovista.psu.edu/CrimeViz/.

where crime does not actually occur. Further, we found that the most intuitive way to present advanced interaction controls was through a layout organized by Peuquet's (1994) TRIAD of spatial, temporal, and attribute information, resulting in three distinct interface panels. We also implemented a time wheel filtering interface to speed cyclical temporal queries, an idea first described by Edsall and Peuquet (1997), and accelerator shortcuts for recurring temporal queries (e.g., weekend versus weekday, daytime versus nighttime versus rush hour).

We selected a journal that does not enforce a maximum word limit (Roth, Ross, MacEachren 2015) so that we could comprehensively report all details regarding process, methods, and design insights from the GeoVISTA CrimeViz UCD study. In hindsight, we should have posted source code and video demonstrations as part of the contribution, particularly considering that GeoVISTA CrimeViz is no longer functional online. Archived code and videos remain available through the project website: https://www.geovista.psu.edu/CrimeViz/.

Our user-centered approach led to excellent buy-in and uptake at the Harrisburg Bureau of Police, and the goodwill generated through the deep partnership allowed us to run a summative controlled experiment on interactive cartography. The summative controlled experiment was separate from the planned UCD case study, but used GeoVISTA CrimeViz as a "living laboratory". To impose some control, we asked participants to complete fifteen benchmark tasks (space + time + 
attribute information operands $\times$ identify + compare + rank + associate + delineate user objectives) derived from my dissertation work on interaction operators (pan, zoom, search, filter, etc.). The summative controlled experiment resulted in a number of generalizable interaction design insights: user objectives increase in cognitive sophistication from identify $\rightarrow$ compare $\rightarrow$ rank $\rightarrow$ associate $\rightarrow$ delineate, the search operator is useful for simple objectives like identify but the filter operator becomes more important for complex objectives like delineate, and there are a number of suboptimal operator + objective strategies that should be prevented in cartographic interface design (see Roth, MacEachren 2016 for details).

Our work with the Harrisburg Bureau of Police did result in several interesting insights into the nature of criminal activity and public safety. For instance, we found that arsons peak on Wednesday and generally follow a bell curve centered midweek with far fewer incidents on the weekend. We also confirmed that added foot patrol in summer months within Harrisburg effectively reduced violent crime in the city center. However, I think we missed an opportunity to report design insights about social justice. There were a number of mapping features that we discussed during the UCD study that were not implemented in the tool for fear of presenting misleading correlations with demographic information that might reinforce rather than resolve racial and socioeconomic disparities in policing, arrests, and convictions. Because of these discussions about "negative results", we shifted our emphasis during the project from reactive, tactical crime analysis to preventative, strategic support for administration to address institutionalized issues regarding crime and social inequalities. Lessons learned from the UCD study implicitly influence my teaching of research and designs ethics today, but we failed to report these negative results in previous publications.

\subsection{The NOAA Lake Level Viewer}

The NOAA (National Oceanic and Atmospheric Administration, U.S.) Lake Level Viewer is an interactive and web-based visualization supporting adaptive management of future water levels in response to climate change in the Great Lakes region of North America (https://coast.noaa.gov/llv/; Figure 5). The Lake Level Viewer is a sibling visualization to NOAA's Sea Level Rise Viewer covering the Atlantic, Pacific, and Gulf coasts of the U.S., a visualization that is now in its third release (https://coast.noaa.gov/slr/). The Lake Level Viewer needed a fundamental redesign from the Sea Level Rise Viewer to account for a different Great Lakes problem context: unlike sea levels that are expected to rise across the globe, warmer temperatures in the Great Lakes suggest less winter surface ice, leading to greater evaporation particularly in the headwaters of Lake Superior, and ultimately reduced lake levels across the Great Lakes system, although with greater variability from year-to-year 


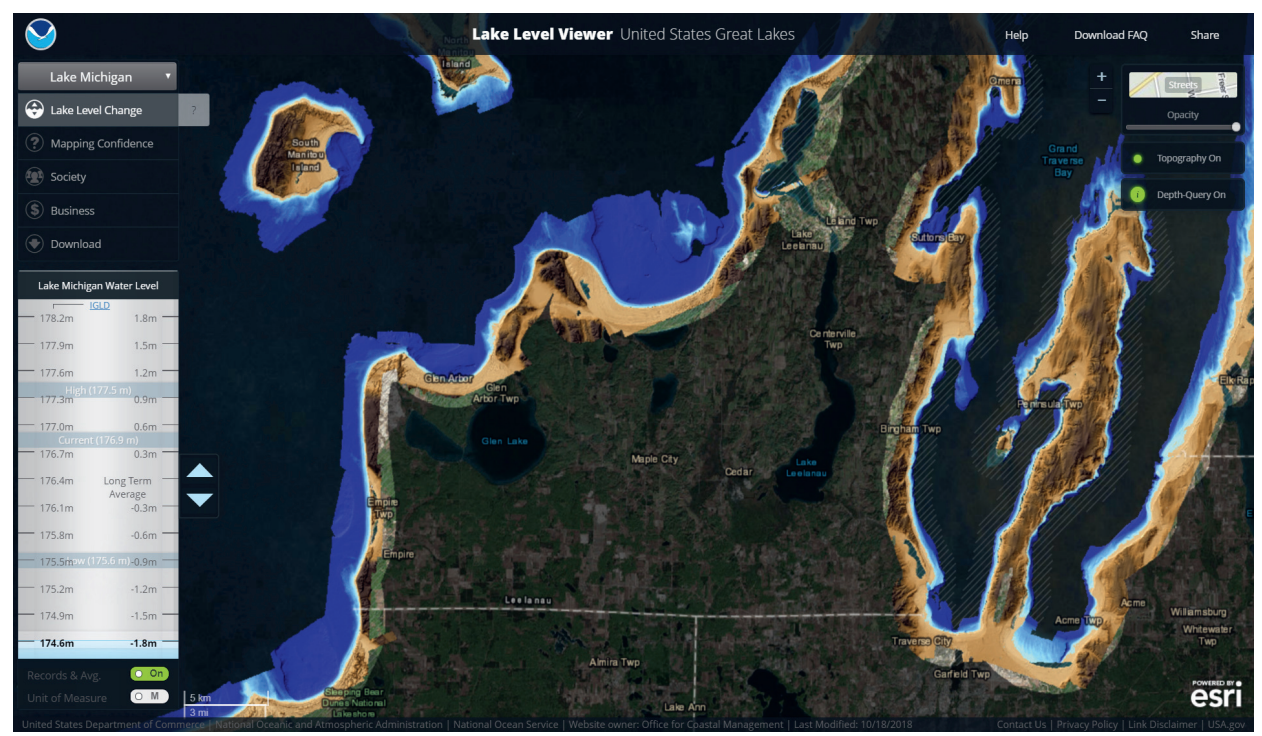

Fig. 5 - NOAA Lake Level Viewer. Source: http://coast.noaa.gov/llv/.

and season-to-season than ocean levels (Angel, Kunkel 2010; Hayhoe et al. 2010). In 2013-2014, I partnered with the University of Wisconsin Sea Grant Institute to plan and facilitate the user-centered dev cycle conducted by NOAA.

Starting with streamlining the UCD process, my experience on the NOAA Lake Level Viewer project was unique in that my team was not responsible for any coding. NOAA contracted us to work with target users to complete conceptual design and prototyping, but then turned to an internal team of developers for the implementation. This was the preferred arrangement given budgetary and time constraints, but it forced the UW team to separate design from development and linearize the design process, a different yet still user-centered approach than the GeoVISTA CrimeViz process. Accordingly, we front-loaded user feedback and mixed our methods: teleconference focus groups for the needs assessment, a competitive analysis of existing water level visualizations to supplement conceptual design, and cognitive walkthroughs with target users on wireframe prototypes. NOAA completed the implementation and transition in less than a month without hiccups, an indication the division of labor was an acceptable compromise.

The competitive analysis served as a discount gap analysis for water level visualization, given the range of tools available today. We analyzed twenty-five visualizations, organizing best practices by five categories of functional needs, divided again by representation versus interaction: (1) representation of the waterline / flood extent, (2) representation of uncertainty in future predictions, (3) representation of basemap and overlay context, (4) supported interaction 
operators, and (5) underlying interactive web mapping technologies. A key observation from the domain gap analysis was that no existing visualizations supported decreasing fresh water levels given the emphasis on sea level rise, requiring our team to explore novel cartographic solutions for visualizing exposed in addition to inundated land (see Roth, Quinn, Hart 2015 for details).

The primary scholarly contribution from the NOAA Lake Level Viewer project was our adapted use of wireframes to elicit user feedback before implementation. We separated representation versus interaction design into two sets of wireframes: high-fidelity wireframes using real lidar-derived bathymetry data to evaluate our proposed representation solutions and low-fidelity wireframes using simple sketches to assess our proposed interaction solutions. We then asked 18 target users distributed across the Great Lakes region to complete a cognitive walkthrough with the wireframes in which they assumed target use case scenarios and imagined how they would make use of the visualization to achieve associated goals. While a long-established method in usability engineering (e.g., Polson et al. 1992), cognitive walkthroughs rarely are administered to evaluate maps or visualizations. The cognitive walkthroughs confirmed that wireframes can be used in place of functional releases to save project resources and time, generating a number of methodological insights for employing wireframes in a streamlined user-centered dev cycle (see Roth et al. 2017 for details).

The cognitive walkthroughs also revealed a number of potentially transferable insights. For instance, assessment of high-fidelity wireframes helped us identify the optimal color ramp for depicting both inundated and exposed land. Because each of the Great Lakes is at a different elevation from sea level, we clarified the entry point to the visualization by adding a splash page requiring users to select a specific lake before proceeding. We reorganized the interface controls so that configuration tools (i.e., used before exploring the map) were on the left of the layout and context tools (i.e., used after interpreting the map) were on the right, following Western reading conventions. We also redesigned the water level slider bar as a vessel that empties and fills, evoking a visual metaphor for intermittent exposure and inundation instead of one-directional "rise". Finally, we summarized a number of "wishlist" items - such as relating design to IPCC climate change scenarios and providing additional local context about the impacted people and places - for NOAA stakeholders that were not implemented in the Lake Level Viewer, but ultimately were included in the third release of the Sea Level Rise Viewer. The improved Sea Level Rise Viewer illustrates the value of documenting "negative" transferable insights from UCD studies, as ideas not possible on the current project nonetheless illuminated new pathways for future research and design.

The divided, compromise process did limit the overall contribution of the NOAA Lake Level Viewer UCD study in several ways. Because we were not involved in 
implementation and transition, we were not included in final decisions regarding design and technology and thus were unable to report on a comprehensive UCD case study. Because we did not develop the tool ourselves, we were unable to archive a repository of the source code. A code archive is not available through NOAA, although $\pm 6 \mathrm{ft}$ water level tilesets are available for reuse in other web maps. This is a particularly troubling issue given U.S. presidential initiatives to delete climate-related data products from federal websites, although at the time of writing, the Lake Level Viewer is not impacted by the broader U.S. political context. Finally, because of the handoff midway through the UCD process, we did not gain the opportunity to perform a summative controlled experiment.

Perhaps my biggest disappointment with the NOAA Lake Level Viewer UCD study was our failure to contribute to climate change science in a meaningful way. We did learn a great deal about future climate conditions in the Great Lakes through the project, but were discouraged from sharing these insights about the regional geography of climate change during the peer-review process (i.e., by cartographers and not climate scientists or the U.S. president). Perhaps because climate change is a pressing - and to some political - geographic issue, we were instructed to "stick to what we know" and downplay topics perceived to fall outside of our training or expertise. This experience points to a misconception of UCD studies that the contribution is solely about making products and not also about the people or places impacted by their use, a tension I am still uncertain how best to resolve moving forward.

\subsection{Global Madison}

Global Madison is a responsive, mobile web map supporting teaching and learning about globalization using the streets of Madison (WI, U.S.) as a situated classroom (http://www.geography.wisc.edu/globalmadison/; Figure 6). Global Madison supports the International Studies 101 course at the University of Wisconsin-Madison, a three-credit, freshman-level course that explores global relationships and disparities regarding finance, governance, health, and trade, among others. The IS101 course enrolls 400 students and draws a large number of international students given the topic. I partnered with the IS101 instructor in my capacity with the University of Wisconsin Cartography Lab to design a mobile-first mapping website rooting discussion of globalization in local context, using Global Madison first to guide students on a walking tour using their device GPS and then to deliver multimedia content and critical thinking prompts at historic landmarks within the city. Further, the UCD study was conceptualized and executed as part of a 2014 graduate seminar on mobile map design, enlisting both cartography and geography students in the design and development process. 


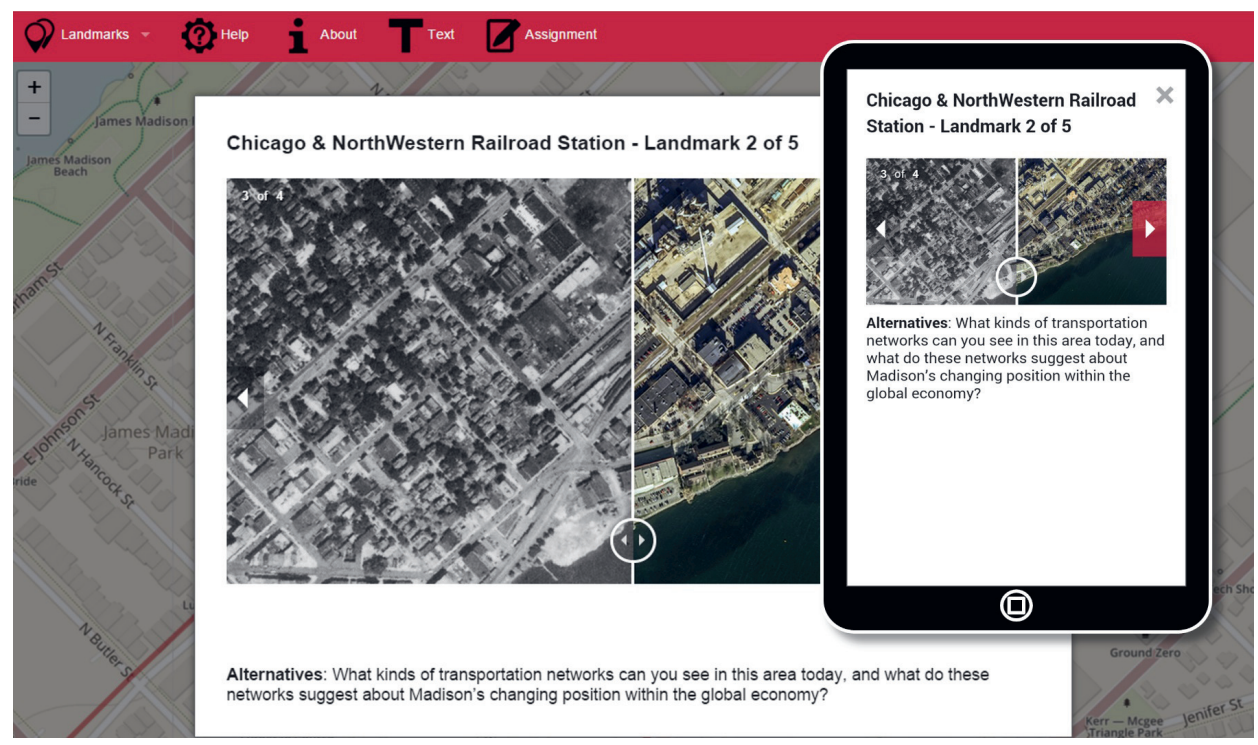

Fig. 6 - Global Madison. Source: http://www.geography.wisc.edu/globalmadison/.

We began the Global Madison UCD study with needs assessment interviews of the instructor and teaching assistants in the IS101 course. IS101 uses the Sparke (2012) Introduction to Globalization textbook, which defined much of the domain problem context for the primarily educational product. Instead, we used the seminar to complete a comprehensive literature review on the state-of-design in mobile mapping. We organized the literature review into seven categories: (1) map composition and layout, (2) scale and generalization, (3) projection, (4) symbolization, (5) typography, (6) map elements (\#1-6 covering representation), and (7) interaction (to complement representation) (see Ricker, Roth 2018 for expanded discussion). Thus, while we did not perform a gap analysis on the problem domain of globalization, we did use the UCD study to organize an emerging area of design within cartography, an alternative potential contribution at the needs assessment stage of UCD.

We then split design into three teams working in parallel: the storytelling team, the UI/UX team (user interface / user experience design), and the evaluation team. Delineation into teams allowed students to self-select their seminar responsibilities based on interests and skills: the storytelling team was responsible for planning the route and packaging archival research on historic sites into compelling short stories and prompts, the UI/UX team was responsible for planning the responsive design strategy and implementing the application on the Open Web Platform, and the evaluation team was responsible for managing the UCD process and administering the user studies. Team leaders gave weekly progress updates, 
adding feedback loops to the UCD process to discuss crosscutting issues. Thus, the Global Madison parallelized process was the conceptual inverse to the NOAA Lake Level Viewer linearized process, despite both assigning iterative milestones to different teams. While parallelized teams were ideal for the academic seminar setting, self-selection into teams on a project-by-project basis in non-academic settings also has process advantages, such as giving agency to employees over the design process, enabling employees to add new skills and experience, and combatting fatigue and burnout on specific design tasks.

We decided on a mixed-method evaluation pairing an online survey with 244 students with a field observation with 18 students. This combination proved valuable, as the online survey captured broad impressions about the selected route and landmarks, the responsive design strategy used for the mobile map, and the overall situated learning experience. Importantly, the broad online survey also captured individual differences in opinions and experiences by age, gender, nationality, and language, important considerations for educational and public-facing maps and visualizations designed to work for diverse audiences. The field observation then provided a deeper look at the mobile map design, identifying specific design solutions for broad feedback in the online survey and identifying several additional "critical" incidents that were infrequently reported in the survey, but found to severely impact the guided tour when observed in-person.

The mixed-method evaluation resulted in a number of transferable insights for mobile maps. First, we focused our design revisions on critical issues that might leave users stranded during the guided tour, particularly reducing emphasis on multimedia features that consumed excessive battery life. Second, we appended the GPS-based routing with an offline cache and downloadable static route maps, both in case data connectivity dropped. Third, we found that we needed to provide a more consistent feed of educational content, given the 2.5-hour planned length of the guided tour, and to associate this content with salient landmarks to draw easier connections between map and landscape. Finally, we observed three critical incidents when users ignored crossing signals or walked in front of traffic, leading us to add information alerts to promote user safety given the assumed mobility.

The comprehensive Global Madison case study is available in Roth et al. (2018). We archived source code for Global Madison as well as the online survey and field observation protocols as supplemental materials with the publication, providing full provenance. We did not conduct a summative controlled experiment using Global Madison, although one is planned as part of future maintenance.

Unlike the GeoVISTA CrimeViz and, especially, the NOAA Lake Level Viewer UCD studies, we foregrounded geographic contributions in the Global Madison UCD study and write-up. We gave our archival research into Madison's relationship to global inequities, interdependencies, and alternatives substantial space in the resulting publication as "Case Study". Further, our design and evaluation loops 
did not just address mobile map design, but also situated learning with these mobile maps, a critical geographic pedagogy that requires students to engage with place-based topics and issues under realistic cultural, logistical, and technological conditions (Anderson, Reder, Simon 1996). Upon reflection, I suspect one reason for the emphasis on geographic as well as cartographic insights in the Global Madison UCD case study was having an academic partner and educational use case, making it easier to view the project as a collaboration rather than a contract as all invested members saw research and publication as important outcomes in addition to design and development of a single product.

\section{Summary and outlook}

In this essay, I provide a partial answer to the question: How do user-centered design studies contribute to cartography? Expanding upon Sedlmair, Meyer and Munzner (2012), Robinson et al. (2005), and others, I argue there are at least eight moments during design studies when the designers can slow the process and enhance the overall contribution to cartography: (1) domain gap analyses, (2) adapted or novel user-centered methods, (3) streamlined user-centered design processes, (4) transferable design insights, (5) comprehensive user-centered design case studies, (6) novel or unique maps and visualizations, (7) summative controlled experiments, and (8) new insights into pressing geographic problems. Thus, a scholarly manuscript on a UCD study must include at least one but better several of these contributions to warrant publication. I then applied this analytical framework to my own past collaborative work in a retrospective analysis of three UCD case studies, illustrating the different kinds of scholarly contributions and noting several missed opportunities therein.

As evinced from the prior discussion, I am a strong advocate for UCD studies and their contributions. For design-as-practice, UCD studies result in better maps and visualizations, typically saving time and resources in the process. For research scholarship, UCD studies offer transferable insights, and, if planned and executed with intention, can generate profound cartographic contributions to context, method, process, design, provenance, product, basic science, and, perhaps most importantly, do good. Much future work is needed to enrich the proposed analytical framework, both with additional UCD case studies and expanded understandings of how UCD studies contribute. One feasible starting point is to identify viable, cost-effective solutions for the key concerns listed in Table 2.

In summary, do you want to make maps or do you want to research maps? I say do both! 


\section{References}

ANDERSON, J.R., REDER, L.M., SIMON, H.A. (1996): Situated learning and education. Educational researcher, 25, 4, 5-11.

ANGEL, J.R., KUNKEL, K.E. (2010): The response of Great Lakes water levels to future climate scenarios with an emphasis on Lake Michigan-Huron. Journal of Great Lakes Research, 36, 51-58.

BARKHUUS, L., RODE, J.A. (2007): From mice to men-24 years of evaluation in CHI. Paper read at Proceedings of the SIGCHI Conference on Human Factors in Computing Systems.

BORKIN, M.K., GAJOS, A., PETERS, D., MITSOURAS, S., MELCHIONNA, F., RYBICKI, C., FELDMAN, PFISTER, H. (2011): Evaluation of artery visualizations for heart disease diagnosis. IEEE transactions on visualization and computer graphics, 17, 12, 2479-2488.

BRUGGMANN, A., FABRIKANT, S.I. (2016): How does GIScience support spatio-temporal information search in the humanities? Spatial Cognition \& Computation, 16, 4, 255-271.

BUCKINGHAM, T.M.A., ed. (2019): Map Production.

BUTTENFIELD, B. (1999): Usability evaluation of digital libraries. Science \& Technology Libraries, 17, 3/4, 39-59.

ÇÖLTEKIN, A., HEIL, B., GARLANDINI, S., FABRIKANT, S.I. (2009): Evaluating the effectiveness of interactive map interface designs: a case study integrating usability metrics with eye-movement analysis. Cartography and Geographic Information Science, 36, 1, 5-17.

CRAMPTON, J.W. (2011): Mapping: A critical introduction to cartography and GIS: John Wiley \& Sons.

DAVIES, C. (1998): Analysing 'work' in complex system tasks: An exploratory study with GIS. Behaviour and Information Technology, 17, 4, 218-230.

EDSALL, R., PEUQUET, D. (1997): A graphical user interface for the integration of time into GIS. Paper read at Proceedings of the 1997 American congress of surveying and mapping annual convention and exhibition, Seattle, WA.

ELWOOD, S., LESZCZYNSKI, A. (2018): Feminist digital geographies. Gender, Place \& Culture, 1-16.

GABBARD, J.L., HIX, D., SWAN, J.E. (1999): User-centered design and evaluation of virtual environments. IEEE Computer Graphics and Applications, 19, 6, 51-59.

GREENBERG, S., BUXTON, B. (2008): Usability evaluation considered harmful (some of the time). Paper read at Proceedings of the SIGCHI conference on Human factors in computing systems.

GRIFFIN, A., ÇÖLTEKIN, A., CHRISTOPHE, S. (2018): Reproducibility in cartography. Paper read at International Cartographic Association (ICA) Joint Commission Workshop on Reproducibility in Cartography, 27 April, at Olomouc, Czechia.

GRIFFIN, A. L., WHITE, T., FISH, C., TOMIO, B., HUANG, H., SLUTER, C.R., BRAVO, J.V.M., FABRIKANT, S.I., BLEISCH, S., YAMADA, M. (2017): Designing across map use contexts: a research agenda. International Journal of Cartography, 3, Supl. 1, 90-114.

GRINSTEIN, G., KOBSA, A., PLAISANT, C., SHNEIDERMAN, B., STASKO, J.T. (2003): Which comes first, usability or utility? Paper read at $14^{\text {th }}$ IEEE Visualization (VIZ'03), 22-24 October, at Seattle, WA.

HAKLAY, M., NIVALA, A.M. (2010): User-centered design. In: Haklay, M. (ed.): Interacting with geospatial technologies. Wiley-Blackwell, West Sussex, UK, 91-106.

HAYHOE, K., VANDORN, J., CROLEY, T., II, SCHLEGAL, N., WUEBBLES, D. (2010): Regional climate change projections for Chicago and the US Great Lakes. Journal of Great Lakes Research, 36, 7-21. 
ISENBERG, T., ISENBERG, P., CHEN, J., SEDLMAIR, M., MÖLLER, T. (2013): A systematic review on the practice of evaluating visualization. IEEE transactions on visualization and computer graphics, 19, 12, 2818-2827.

KITCHIN, R., DODGE, M. (2007): Rethinking maps. Progress in Human Geography, 31, 3, 331-344.

KLIPPEL, A., WEAVER, ROBINSON, A.C. (2011): Analyzing cognitive conceptualizations using interactive visual environments. Cartography and Geographic Information Science, 38, 1, 52-68.

KOH, L.C., SLINGSBY, A. DYKES, J., KAM, T.S. (2011): Developing and applying a user-centered model for the design and implementation of information visualization tools. Paper read at Information Visualisation, (IV), $15^{\text {th }}$ International Conference on.

KOSARA, R., HEALEY, C.G., INTERRANTE, V., LAIDLAW, D.H., WARE, C. (2003): User studies: Why, how, and when? IEEE Computer Graphics and Applications, 4, 20-25.

KRUG, S. (2000): Don't make me think: A common sense approach to web usability. $2^{\text {nd }}$ ed., New Riders Publishing, Berkeley.

LAM, H., BERTINI, E., ISENBERG, P., PLAISANT, C., CARPENDALE, S. (2012): Empirical studies in information visualization: Seven scenarios. IEEE transactions on visualization and computer graphics, 18, 9, 1520-1536.

LLOYD, D., DYKES, J. (2011): Human-centered approaches in geovisualization design: Investigating multiple methods through a long-term case study. IEEE transactions on visualization and computer graphics, 17, 12, 2498-2507.

MACEACHREN, A.M., KRAAK, M.J. (2001): Research challenges in geovisualization. Cartography and Geographic Information Science, 28, 1, 3-12.

MOLICH, R., NIELSEN, J. (1990): Improving a human-computer dialogue. Communications of the ACM, 33, 3, 338-348.

MUNZNER, T. (2009): A nested process model for visualization design and validation. IEEE Transactions on Visualization \& Computer Graphics, 6, 921-928.

NIELSEN, J. (1992): The usability engineering life cycle. Computer, 25, 3, 12-22.

NORMAN, D. (2013): The design of everyday things. Revised and expanded edition: Constellation. Basic books, New York.

OOMS, K., SKARLATIDOU, A., eds. (2018): Usability engineering and evaluation.

PEUQUET, D.J. (1994): It's about time: A conceptual framework for the representation of temporal dynamics in geographic information systems. Annals of the Association of american Geographers, 84, 3, 441-461.

POLSON, P.G., LEWIS, C., RIEMAN, J., WHARTON, C. (1992): Cognitive walkthroughs: a method for theory-based evaluation of user interfaces. International Journal of Man-Machine Studies, 36, 5, 741-773.

RICKER, B., ROTH, R. (2018): Mobile Maps and Responsive Design. Geographic Information Science \& Technology Body of Knowledge:CV 40.

ROBINSON, A.C., CHEN, J., LENGERICH, E.J., MEYER, H.G., MACEACHREN, A.M. (2005): Combining usability techniques to design geovisualization tools for epidemiology. Cartography and Geographic Information Science, 32, 4, 243-255.

ROBINSON, A.C., WEAVER, C. (2006): Re-visualization: Interactive visualization of the process of visual analysis. Paper read at Workshop on Visualization, Analytics \& Spatial Decision Support at the GIScience conference.

ROONEY, C., BEECHAM, R., DYKES, J., WONG, W. (2017): Dynamic Design Documents for supporting applied visualization. IEEE VIS 2017. 
ROSSON, M.B., CARROLL, J.M. (2002): Scenario-based Design. In: Jacko, J., Sears, A. (eds.): The human-computer interaction handbook: Fundamental evolving technologies and emerging applications, Lawrence Erlbaum Associates, Hillsdale, NJ, 1032-1050.

ROTH, R., YOUNG, S., NESTEL, C., SACK, C., DAVIDSON, B., JANICKI, J., KNOPPKEWETZEL, V., MA, F., MEAD, R., ROSE, C. (2018): Global Landscapes: Teaching globalization through responsive mobile map design. The Professional Geographer, 1-17.

ROTH, R.E., ÇÖLTEKIN, A., DELAZARI, L., FILHO, H.F., GRIFFIN, A., HALL, A., KORPI, J., LOKKA, I., MENDONÇA, A., OOMS, K. (2017): User studies in cartography: opportunities for empirical research on interactive maps and visualizations. International Journal of Cartography, 3, Supl. 1, 61-89.

ROTH, R.E., FINCH, B.G., BLANFORD, J.I., KLIPPEL, A., ROBINSON, A.C., MACEACHREN, A.M. (2011): Card sorting for cartographic research and practice. Cartography and Geographic Information Science, 38, 2, 89-99.

ROTH, R.E., HART, D., MEAD, R., QUINN, C. (2017): Wireframing for interactive \& webbased geographic visualization: designing the NOAA Lake Level Viewer. Cartography and Geographic Information Science, 44, 4, 338-357.

ROTH, R.E., MACEACHREN, A.M. (2016): Geovisual analytics and the science of interaction: an empirical interaction study. Cartography and Geographic Information Science, 43, 1, $30-54$.

ROTH, R.E., QUINN, C., HART, D. (2015): The competitive analysis method for evaluating water level visualization tools. In Modern Trends in Cartography, Springer, 241-256.

ROTH, R.E., ROSS, K.S., FINCH, B.G., LUO, W., MACEACHREN, A.M. (2013): Spatiotemporal crime analysis in US law enforcement agencies: Current practices and unmet needs. Government Information Quarterly, 30, 3, 226-240.

ROTH, R.E., ROSS, K.S., MACEACHREN, A.M. (2015): User-centered design for interactive maps: A case study in crime analysis. ISPRS International Journal of Geo-Information, 4, 1, 262-301.

SEDLMAIR, M., MEYER, M., MUNZNER, T. (2012): Design study methodology: Reflections from the trenches and the stacks. IEEE Transactions on Visualization \& Computer Graphics, $12,2431-2440$.

SLOCUM, T., CLIBURN, D., FEDDEMA, J., MILLER, J. (2003): Evaluating the usability of a tool for visualizing the uncertainty of the future global water balance. Cartography and Geographic Information Science, 30, 4, 299-317.

SLUTER, C.R., VAN ELZAKKER, C.P., IVÁNOVÁ, I. (2017): Requirements elicitation for geoinformation solutions. The Cartographic Journal, 54, 1, 77-90.

SPARKE, M. (2012): Introducing globalization: Ties, tensions, and uneven integration: John Wiley \& Sons.

ŠTĚRBA, Z., ŠAŠINKA, Č., STACHOŇ, Z., KUBÍČEK, P., TAMM, S. (2014): Mixed research design in cartography: a combination of qualitative and quantitative approaches. Kartographische Nachrichten, 64, 5, 262-269.

TOLOCHKO, R.C. (2016): Contemporary professional practices in interactive web map design, University of Wisconsin, Madison.

TSOU, M.H., CURRAN, J.M. (2008): User-centered design approaches for web mapping applications: A case study with USGS hydrological data in the United States. In: International perspectives on maps and the Internet. Springer, 301-321.

VAN ELZAKKER, C., OOMS, K., KENT, A., VUJAKOVIC, P. (2017): Understanding map uses and users. In The Routledge Handbook of Mapping and Cartography, Routledge, 79-91. 
VAN ELZAKKER, C.P., WEALANDS, K. (2007): Use and users of multimedia cartography. In Multimedia cartography, Springer, 487-504.

WHITE, T. (2017): From Existing Practices to Best Practices: Improving the Quality and Consistency of Participant Assessment Methods in Cartographic User Studies, Geography, Kansas.

\section{ACKNOWLEDGEMENTS}

I thank Amy Griffin and Petr Kubíček for organizing the special issue and the anonymous reviewers for their feedback. The essay is based on an invited lecture organized by Liqui Meng, Juliane Cron, and Corné van Elzakker as part of the Erasmus Mundus MSc Cartography program and later presented at the 2018 ICA Joint Commission Workshop in Olomouc, Czechia, organized by Vít Voženílek and Palacký University. Portions of this research were funded by VACCINE Award \#2009-ST-061-CI0001, NOAA Award \#167152, and NSF CAREER Award \#1555267.

The content of this Special Issue of Geografie Journal has been prepared together with the International Cartographic Association, namely with the Commission on Cognitive Issues in Geographic Information Visualization, the Commission on Use, User and Usability Issues, and the Commission on Atlases. Selected authors were contacted during the ICA Commissions' joint workshop "Atlases, Cognition, Usability" held in Olomouc (Czechia) at Palacký University in April 2018. The content of the Special Issue reflects the current topics handled by the Commissions and we hope that the special issue increases awareness and knowledge of cognitive and usability issues with geographic information among the broader geographic community. Preliminary selection of manuscripts and pre-submission communication with authors was done by Petr Kubíček (Masaryk University, proposed incoming co-Chair of the Cognitive Issues in Geographic Information Visualization Commission) and Amy Griffin (RMIT University, current co-chair of the Cognitive Issues in Geographic Information Visualization Commission). We thank all of the authors who contributed their research to this special issue. 